\title{
L'eau et l'argent à Potosi (ancien Haut-Pérou puis Bolivie)
}

\author{
par Alain Gioda \\ ORSTOM-SENAMHI, Cochabamba, Bolivie \\ et \\ Carlos Serrano \\ Universidad Autónoma Tomás Frías, Potosi, Bolivie
}

avec la collaboration de

Markus Frey

Étudiant à l'Albert-Ludwig Universität, Fribourg, Allemagne

The mining city of Potosi located in the arid zone of the Andes range has an exceptional heritage of industrial archeology with a factory of silver mineral trituration, its mill and other hydraulic installations. However, the Potosi particularity is to keep efficient the water network built by the Spaniands between the XVIth and the XVIIth centuries and especially a complicated tank system of twenty lakes. The Viceroy Francisco de Toledo ordered in 1572 these pharaonic works - the tanks are located between $4300 \mathrm{~m}$ and $4800 \mathrm{~m}$ above sea level - to adapt the production of silver to the diminution of the quality of the veins and so Toledo pushed to adopt an advanced technology coming from Mexico which needed important and permanent quantities of water : the "patio". So, all trituration factories - more than eighty in 1633 - were located close to the artificial channel, the so-called Ribera de la Vera Cruz, which has been crossing the city since 1575. The Ribera became the economical center of the richest city in the World and the most populated town of America at the beginning of the XVIIth century (160 000 inhabitants in 1611). This hydraulic masterpiece was completely restored by the North-American engineer William E. Rudolph in the thirties to provide huge quantities of water to the factories during the tin mining boom. Actually, the Ribera still crosses Potosi and its water is used by thirty small factories of lead, silver, zinc, and tin.

\section{Avant-propos [par A. Goubet]}

L'énergie hydraulique, qui n'a été baptisée "houille blanche" que vers 1900 sous sa forme hydroélectrique, a joué un rôle considérable depuis près de 2000 ans (à l'époque, elle n'était concurrencée que par le vent et les animaux - ou les esclaves).

Déjà maîtrisée par les Romains, la technique a dû subsister pendant tout le Haut Moyen Age pour exploser en Europe à partir du Xe siècle.

Des villes se sont développées en France autour de sites favorables : une rivière de plaine présentant un "rapide", c'est-à-dire une dénivellation de quelques mètres permettant l'installation en cascade de deux ou trois moulins, la rivière étant elle-même partagée en deux ou trois bras (par exemple Troyes, Chartres, peut-être Evreux).

Le développement de Potosi, traité dans le présent article est une autre brillante illustration du rôle de l'énergie hydraulique... à la différence près que dans ce dernier cas, l'homme a même dû créer la rivière.

L'article met aussi en évidence le caractère de la colonisation espagnole qui a "exporté" largement ses techniciens pour exploiter les filons de métaux précieux.

\section{I $\square$ INTRODUCTION}

En partant des traces actuelles du passé industriel, la métallurgie et la houille blanche de l'époque coloniale (1545-1825) seront analysées grâce à des sources documentaires. Les informations tirées des publications de Capoche $(1547-\div 1613$ ?), Arzáns $(1676-\uparrow 1736)$ et Cañete (1754†1816) seront complétées et corrigées par des documents d'archives inédits de mineurs et techniciens. Au cours du temps, le nombre des usines et des machines hydrauliques a souvent varié de même que le degré d'utilisation des eaux des barrages car l'activité des mines d'argent fut cyclique avec cinq boums suivis de longues dépressions entre 1545 et la fin du XIXe siècle. Enfin, seront présentés les travaux de restauration du système hydraulique antique au XXe siècle.

\section{II $\square$ UN MILIEU ARIDE ET FROID POUR UNE CITÉ-MUSÉE}

Potosi compte aujourd'hui environ 120000 habitants. Aux limites de l'Altiplano méridional et des vallées interandines, elle est bâtie à $3980 \mathrm{~m}$ d'altitude sur les flancs du Cerro Rico (4 $890 \mathrm{~m}$ ), la montagne d'argent (Fig. 1). Le climat y 


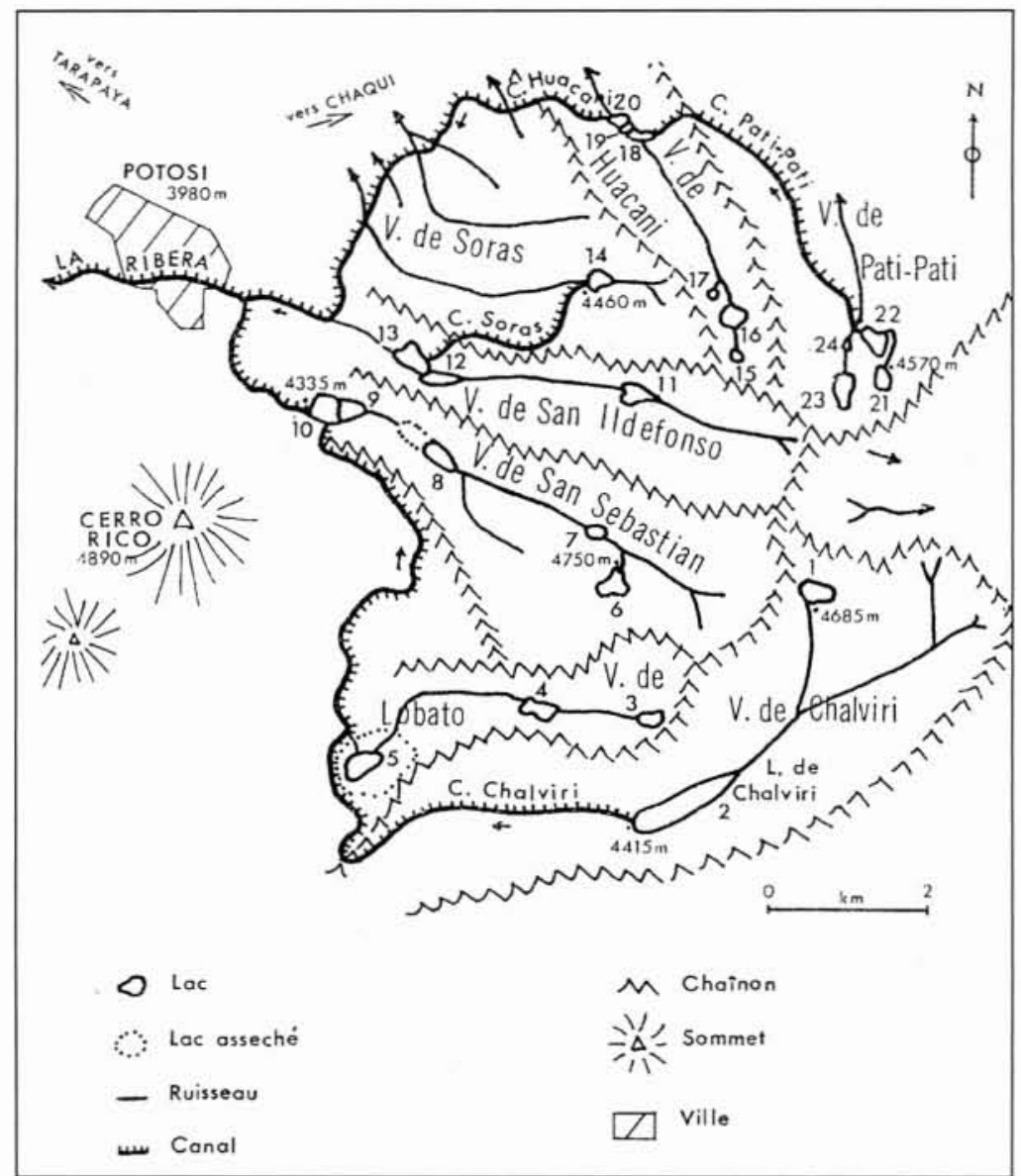

1. Localisation des lacs coloniaux de Potosi dans six vallons (V.) situés à l'est de la montagne d'argent, le Cerro Rico. Schéma du réseau d'adduction et des barrages hérités de la période coloniale (d'après un cadre de Gaspar M. Berrío de 1758 des Musées Universitaires de Sucre et Rudolph [15], modifiés). La numérotation des lacs est la même que dans le tableau 1. Les coordonnées de Potosi sont les suivantes : lat. $19^{\circ} 34^{\prime} 18^{\prime \prime} \mathrm{S}$. ; long. $65^{\circ} 34^{\prime} 25^{\prime \prime} \mathrm{O}$. est froid et aride avec 142 jours de gel par an, une température moyenne de $8,9^{\circ} \mathrm{C}$ et $406 \mathrm{~mm}$ par an de précipitations, valeurs calculées sur la période 1958-1994. Toutefois, Potosi est aussi une cité-musée de la période coloniale espagnole, classée par l'Unesco patrimoine de l'humanité. Parmi les richesses de Potosi, sont conservées dans le domaine de l'archéologie industrielle : des machines hydrauliques, des ruines d'usines (Fig. 2), un château d'eau de la fin du XVIIIe siècle, une vingtaine de lacs de barrages bâtis à partir de 1573 (Fig. 3), leur émissaire, le canal de la Ribera, etc. Entre décembre 1995 et novembre 1996, la coopération espagnole a financé la restauration de la partie hydraulique de l'usine San Marcos de concassage de minerai d'argent, installée sur la Ribera en pleine ville dans la rue $\mathrm{La} \mathrm{Paz}$ (Fig. 4).

Potosi naquit spontanément en 1545 de la découverte du plus grand gisement argentifere du Monde en 1544. De simple campement minier, en moins de trente ans, Potosi devint une ville-champignon de 120000 habitants en 1572 [1]. La population culmina autour de 160000 personnes entre 1611 et 1650 [6]. C'était la plus grande cité des Amériques, pouvant se mesurer avec le Paris et le Londres de l'époque. Toutefois, Potosi était surtout très riche d'où son surnom de Babylone du Pérou avec 200 tonnes d'argent produites seulement pour l'année 1593. L'expression "Vale un Potosí", popularisée dès le début du XVIIe siècle par le "Don Quichotte" de Cervantès, montre que la monnaie d'argent espagnole frappée dans les Andes fut pendant deux siècles l'équivalent du dollar américain dans les transactions commerciales de l'Occident. Le succès de Potosi dont "l'âge d'or" dura de 1545 à 1650 , soit environ un siècle, s'appuya sur les
2. Perspective des ruines actuelles d'une usine coloniale de la Ribera. L'aqueduc amenant l'eau par le dessus est figuré en noir. Entre deux usines, les canaux de prise installés au fil de l'eau sur la Ribera étaient maçonnés. La dernière partie de l'aqueduc était à peu près construite sur le même schéma pour toutes les usines avec une pente du canal de $0,9 \%$. La roue hydraulique était installée à la fin de l'aqueduc, sur le dessin toujours dans la partie noircie située presque au premier plan. Sachant la pénurie de bois à $4000 \mathrm{~m}$ d'altitude, cette roue a dû être rapidement recyclée pour d'autres usages dès la fin de l'utilisation de la houille blanche au XIXe siècle. D'autres levés topographiques de ruines d'usines viennent d'être publiés [21].

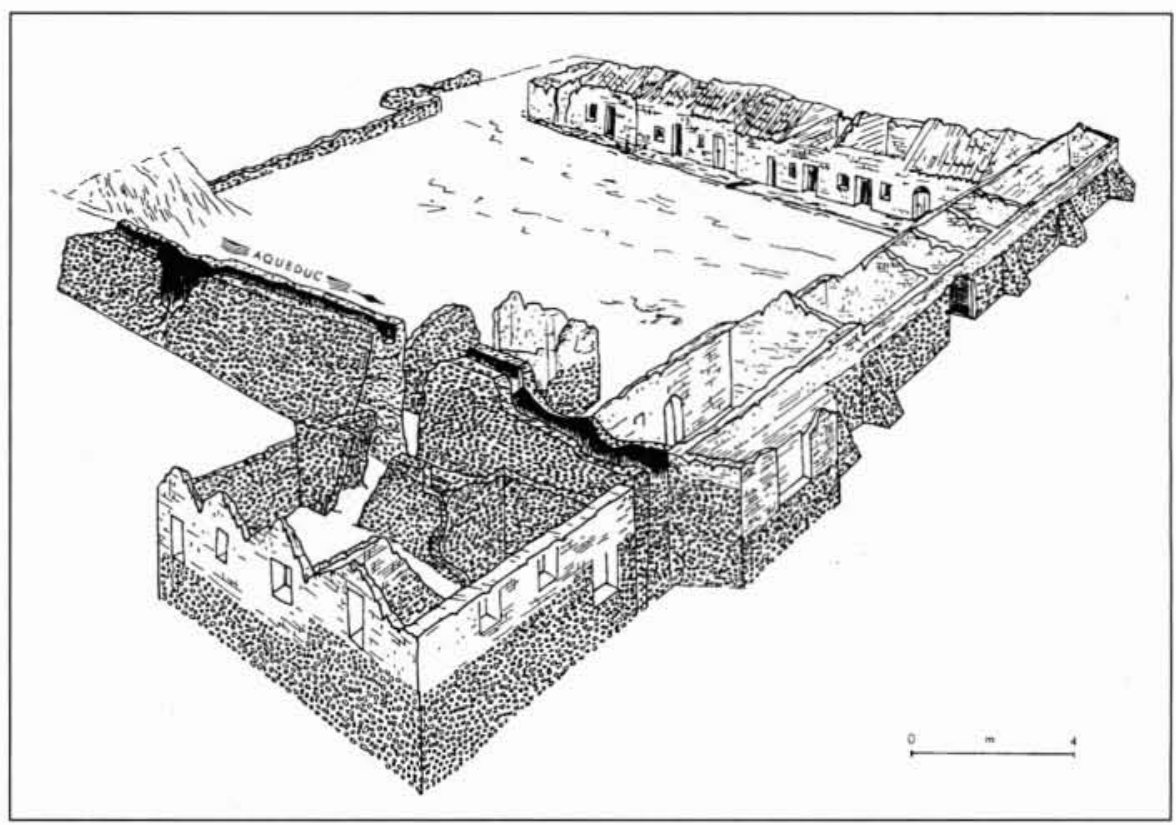




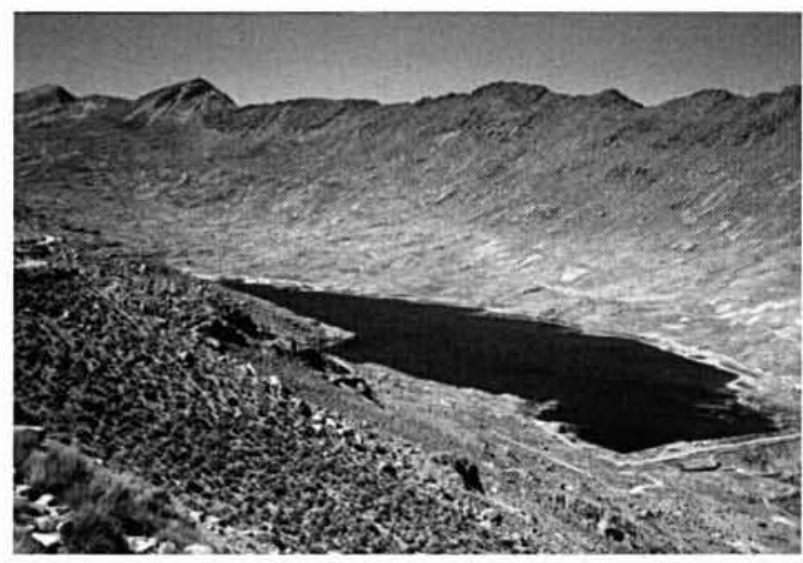

3. Le lac de Chalviri à $\mathbf{4 4 1 5} \mathbf{m}$ d'altitude, le plus grand de la période coloniale de Potosi, qui peut atteindre près de 3 millions de $\mathrm{m}^{3}$ de réserve après les bonnes saisons en mars-avril. La plupart des précipitations se font sous forme de grêle à cette altitude. Au temps de la colonie, la capacité de la retenue était deux fois moindre.

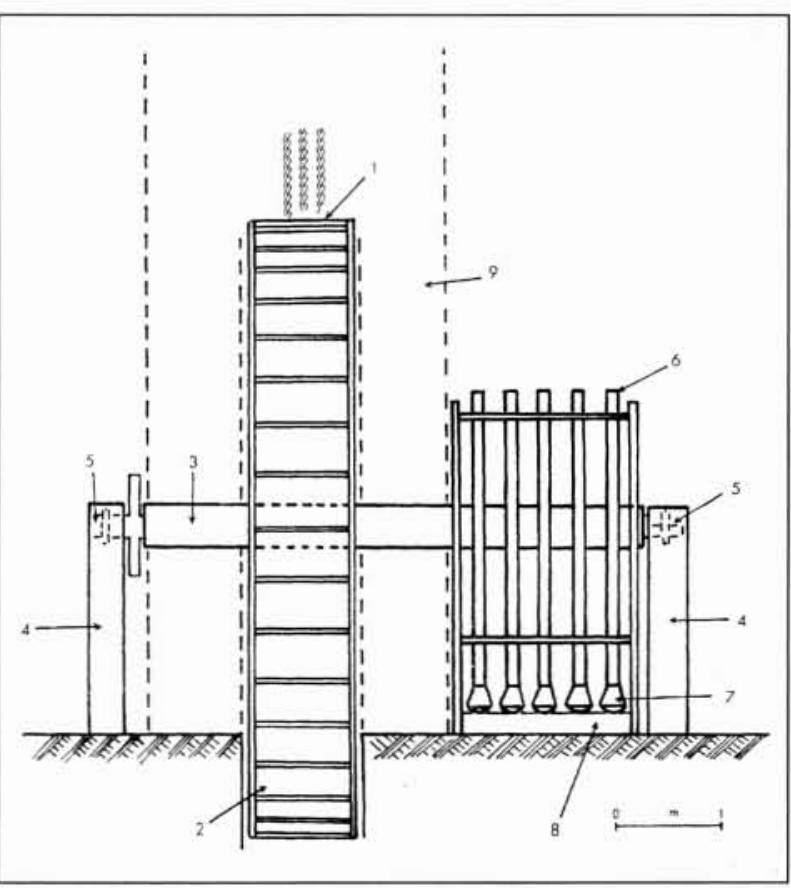

4. Vue de face de la roue hydraulique et du concasseur de l'usine San Marcos sur la Ribera de Potosi depuis sa restauration en 1995-96 (d'après Serrano [19]).

1) Roue ; 2) Aube ; 3) Axe ; 4) Support d'arbre à cames ; 5) Arbre à cames ; 6) Pilon en bois, comme toutes les pièces précédentes au temps de la colonie ; 7) Masse métallique en bronze ou en fer ; 8) Mortier en pierre ; 9) Maçonnerie de l'aqueduc.

Caractéristiques techniques : diamètre de la roue : $5,80 \mathrm{~m}$; largeur : $0,70 \mathrm{~m}$; poids total : 3 tonnes ; nombre de révolutions : 7 à $8 / \mathrm{s}$; débit minimal de l'aqueduc: 5 litres/s; amenée d'eau par le dessus ; hauteur hydrostatique : $8 \mathrm{~m}$. Poids d'un marteau-pilon chaussé d'une masse : $30 \mathrm{~kg}$.

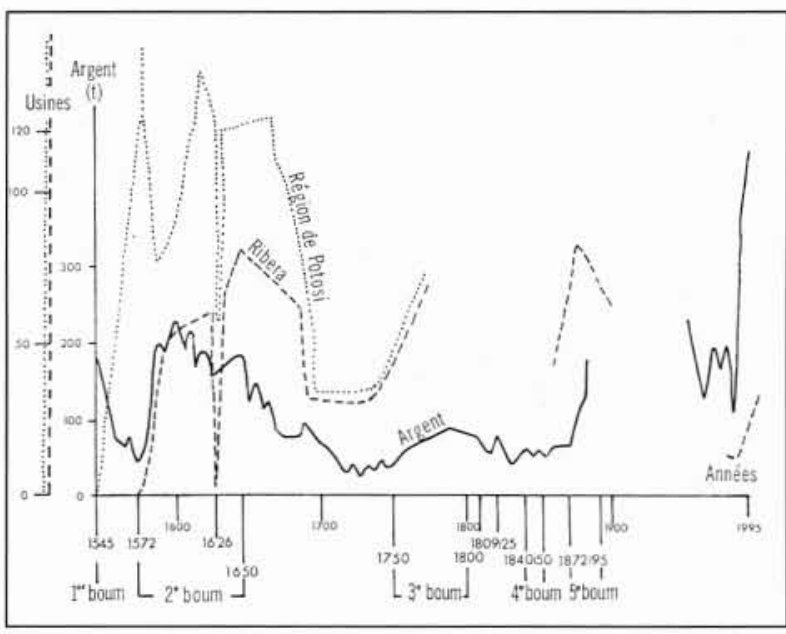

5. L'évolution dans le temps de la production d'argent et du nombre de concasseurs sur la Ribera et dans la région de Potosi. Bakewell [2] est la principale source sur la production d'argent sous la colonie. Le tableau 2 permet de connaître en détail les variations du nombre des installations industrielles.

trois piliers suivants imposés par le vice-roi des Indes Francisco Toledo en 1572 :

a) une révolution technologique dans la métallurgie de l'argent avec un procédé mis au point en 1555, le"patio" (cf. Annexe 1) ;

b) un régime de travaux forcés pour les indiens, seulement aboli en 1812 , la "mita" c'est-à-dire le travail à tour de rôle que fournissaient les communautés indigènes ;

c) une énergie hydraulique abondante, objet de notre travail, qui restera indispensable jusqu'en 1872 , date du début de la modernisation totale des usines avec l'arrivée massive de l'électricité et de la vapeur.

\section{III — L'HYDRAULIQUE DE L'ÉPOQUE COLONIALE (XVIe-XVIIIe SIÈCLES)}

Trois moments importants du Potosi colonial seront présentés grâce à des textes de trois époques différentes et significatives de l'exploitation de l'argent :

- d'abord, "l'âge d'or" de Potosi de 1545 à 1650 qui comporte deux boums, décrit par Capoche en 1583 [4] ;

- puis, la grande crise entre 1650 et 1750 qui correspond au temps de la chronique d'Arzáns rédigée entre 1705 et 1737 [1] ;

- et enfin, la restauration des mines à partir de 1750 et le troisième boum jusqu'à 1800 , illustrés par Cañete dans sa publication de 1787 [3].

\subsection{Capoche et les deux grands boums (1545-1650)}

Luis Capoche, vraisemblablement natif de Séville, était propriétaire de deux usines hydrauliques. Daté de 1583 mais souvent attribué à l'an 1585 , son texte fut dédié au vice-roi des Indes Torrés y Portugal, l'un des successeurs de Toledo [4]. Son propos était de "faciliter la compréhension des thèmes du Cerro [de l'exploitation de l'argent]et ses difficultés". 
En 1566, soit vingt-deux ans après la découverte du grand gisement en 1544 , les petits fours indiens d'origine préincaïque appelés "guayras", installés sur les hauteurs où le vent faisait office de soufflerie, avaient permis l'extraction du minerai d'argent des filons les plus riches et donc le premier boum minier. Mais la production commençait à baisser dangereusement (Fig. 5). Le vice-roi Francisco Toledo, l'administrateur les Indes espagnoles de 1569 à 1581 sous Philippe II, vint à Potosi en 1572 . Il y réunit un groupe représentatif de mineurs pour notamment discuter de l'introduction de la technique du "patio", adaptée à de plus faibles teneurs d'argent, et de la construction de machines hydrauliques. Quatre entrepreneurs s'offrirent pour construire à leurs frais un lac pour récupérer l'eau de pluie et la stocker sachant que la saison des précipitations à Potosi ne dure que trois mois de décembre à février.

\subsubsection{La construction des barrages (à partir de 1573)}

Commencée en 1573 au lac de Chalviri, la construction des barrages mobilisa vingt mille indiens de la "mita" donc soumis aux travaux forcés [1] mais il n'y a plus trace des documents originaux. L'histoire ne semblait pas avoir retenu leurs constructeurs [15]. Seuls étaient connus le vice-roi Toledo, les financiers des travaux et les propriétaires des usines [4, 22]. Toutefois, l'un de nous (C. Serrano) dans les archives de Potosi a retrouvé deux noms de maîtres d'ouvrages hydrauliques : Pedro Sandi et Francisco Ortiz de Aulestia qui donna son nom au lac Ulistia.

Vers 1621, une vingtaine de lacs étaient déjà construits ou aménagés pour un volume total d'environ $6000000 \mathrm{~m}^{3}$ d'eau $[6,15]$ (Tableau 1). Dispersées sur six bassins versants non emboîtés qui couvrent environ $65 \mathrm{~km}^{2}$, les retenues furent interconnectées pour ramener la totalité des eaux dans l'exutoire naturel d'un petit réceptacle naturel d'environ $20 \mathrm{~km}^{2}$ qui recueillait l'écoulement intermittent des deux seuls vallons de San Ildefonso et de San Sebastián (cf. Fig. 1). L'alimentation régulière en énergie hydraulique déterminera en grande partie l'apogée des mines coloniales de Potosi qui correspond au second boum minier (1572-1650).

Selon leur géomorphologie, se distinguent les étendues d'eau suivantes :

- des lacs naturels derrière un verrou glaciaire que les espagnols ont seulement légèrement aménagé (Illimani, Chalviri, Lobato, Providencia, Mazuni, Huacani) ;

- un lac à l'amont d'un verrou morainique et complètement entouré de moraines (Pisco Cocha) ;

- deux lacs dont les moraines frontales étaient détruites

Tableau 1. Les lacs de Potosi dans I'histoire

\begin{tabular}{|c|c|c|c|c|}
\hline Lac & Colonie & Avant & Après & Altitude \\
\hline 1) Illimani & -.. & 0 & 120000 & $4685 \mathrm{~m}$ \\
\hline 2) Chalviri & 1500000 & $1510000+$ & 2900000 & $4415 \mathrm{~m}$ \\
\hline 3) Lobato & $\ldots$ & 0 & 200000 & $4650 \mathrm{~m}$ \\
\hline 4) Ulistia & 300000 & 0 & 360000 & $4610 \mathrm{~m}$ \\
\hline 5) Pisco Cocha & ... & 0 & 340000 & $4490 \mathrm{~m}$ \\
\hline 6) Mazuni & -.. & 0 & 55000 & $4750 \mathrm{~m}$ \\
\hline 7) Cruciza & 150000 & 0 & 47000 & $4605 \mathrm{~m}$ \\
\hline 8) Muñiza & 150000 & 175000 & 175000 & $4365 \mathrm{~m}$ \\
\hline 9) Planilla & 160000 & 90000 & 90000 & $4340 \mathrm{~m}$ \\
\hline 10) San Sebastián & 300000 & 300000 & 300000 & $4335 \mathrm{~m}$ \\
\hline 11) San Fernando & & 0 & 230000 & $4515 \mathrm{~m}$ \\
\hline 12) San Pablo & 300000 & 275000 & 275000 & $4412 \mathrm{~m}$ \\
\hline 13) San Ildefonso & 400000 & 430000 & 430000 & $4410 \mathrm{~m}$ \\
\hline 14) Calderón &.- & 0 & 100000 & $4460 \mathrm{~m}$ \\
\hline 15) Providencia & & 0 & 85000 & $4770 \mathrm{~m}$ \\
\hline 16) Huacani & 300000 & $0++$ & 315000 & $4650 \mathrm{~m}$ \\
\hline 17) Llama-Kunca & & 0 & 11000 & $4450 \mathrm{~m}$ \\
\hline 18) Llama-Miku & -- & 0 & 40000 & $4380 \mathrm{~m}$ \\
\hline 19) Buenaventura & -- & 0 & 12000 & $4375 \mathrm{~m}$ \\
\hline 20) San José & $\ldots$ & 0 & 12000 & $4370 \mathrm{~m}$ \\
\hline 21) Atocha & 150000 & 0 & 160000 & $4570 \mathrm{~m}$ \\
\hline 22) Candeleria & -.- & 0 & 45000 & $4550 \mathrm{~m}$ \\
\hline 23) Santa Lucia & --. & 0 & 130000 & $4590 \mathrm{~m}$ \\
\hline 24) Santa Lucia Chica* & & 0 & 4000 & $4750 \mathrm{~m}$ \\
\hline TOTAL & 6000000 env. & 2780000 & 6456000 & \\
\hline
\end{tabular}

Ce tableau montre le travail de restauration effectué par William E. Rudolph dans les années 1935-36 en le comparant à la situation avant la restauration et fournit une estimation des volumes des lacs lors de la colonie au XVIe-XVIIe siècles (en $\mathrm{m}^{3}$ ). + valeur corrigée des pertes par infiltration

++ pouvait contenir de l'eau mais celle-ci se serait perdue dans le canal d'évacuation en mauvais état

* disparu du temps de la colonie 
6. Dessin original d'Arzáns [1705-37] simplifié montrant le fonctionnement d'une usine d'argent à ciel ouvert et le rôle central de l'eau dans la métallurgie de Potosi au début du XVIIIe siècle avec le concassage du minerai entraîné par deux roues hydrauliques. A gauche (1), un concasseur à une "tête" c'est-à-dire avec les masses disposées d'un seul côté de la roue. A droite (2), un concasseur à deux "têtes" avec les masses des deux côtés de la roue. Au centre (3) étaient les bassins où avait lieu l'amalgamation du minerai d'argent et du mercure (cf. Annexe 1). Les ruines actuelles d'une usine coloniale sont tout à fait similaires (cf. Fig. 2).

par l'érosion et où les espagnols durent construire d'importants barrages avec une longue digue (San Ildefonso, Muniza) [15].

Il y a donc deux types de lacs :

a) en cas de fort surcreusement glaciaire, les Espagnols se limitèrent à la construction de petits barrages et ensuite percèrent un tunnel dans le verrou pour récupérer l'eau à l'aval (Pisco Cocha et Providencia ) ;

b) en cas de moraines frontales à l'aval des grands lacs comme Huacani et Chalviri, la gestion est plus difficile car les infiltrations à travers des remblais sont toujours possibles [15]. Toutefois, un avantage existe : la présence de sable et de cailloux morainiques déjà triés par les anciens glaciers et très utiles pour faire les remblais.

Les Espagnols commencèrent par le lac Chalviri qui a la plus grande capacité. Puis, suivirent dès 1576 l'achèvement de retenues importantes proches de Potosi comme San Sebastián et San Ildefonso [22]. Plus on mettait en service de lacs, plus la production d'argent augmentait en cette période faste. Les grands travaux d'aménagement des barrages peuvent être considérés comme très avancés voire achevés en 1621. Après cette date, on pourrait penser que le nombre des lacs en service ne fera que diminuer car la production d'argent sous la colonie ne retrouva plus jamais son niveau du début du XVIIe siècle. Toutefois, la baisse de la

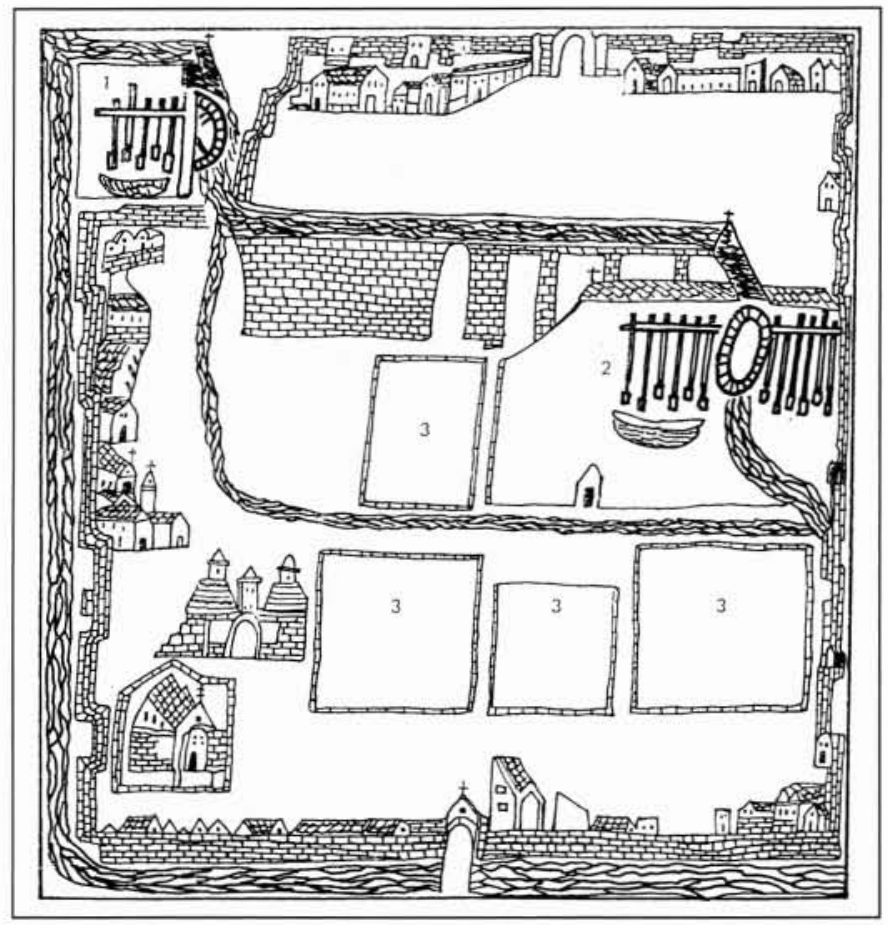

teneur des filons fera que les usines devront traiter de plus en plus de minerai et donc que la demande en eau augmentera d'où une importante restauration des lacs par l'ingénieur Mosquera à la fin du XVIIIe siècle.

\subsubsection{La construction de la Ribera (1574-77)}

Avant la construction des barrages, les usines étaient installées surtout à deux ou trois lieues de la ville - soit 12 à 18 $\mathrm{km}$ car cette unité de mesure valait au Pérou environ $6 \mathrm{~km}$ sur la rivière Cayara au lieu-dit Tarapaya. Toutefois, il fallait y emmener le minerai du Cerro Rico d'où la décision de construire des barrages près de Potosi et de faire traverser la ville par un canal, la Ribera de la Vera Cruz, en exploitant

Tableau 2. L'évolution dans le temps du nombre d'usines à triturer le minerai d'argent de Potosi (d'après Cañete [3], des auteurs cités par ce dernier et des sources antérieures)

\begin{tabular}{|c|c|c|c|c|}
\hline Année & Nombre & Source de l'information & Nombre & $\begin{array}{l}\text { (à gauche, Ribera et autres } \\
\text { localités confondues) }\end{array}$ \\
\hline 1576 & 147 & (Francisco Miguel de Orruño) & 2 & (à droite, seulement usines \\
\hline 1577 & 150 & (vice-roi Toledo) & & $\begin{array}{l}\text { hydrauliques installees sur la } \\
\text { Ribera. }\end{array}$ \\
\hline 1583 & 78 & (Capoche) & 49 & source Carlos Serrano) \\
\hline 1610 & $>140$ & (Nicolas de Guevara et Gaspar Ruiz in Arzáns) & & \\
\hline 1624 & 122 & (marquis de Guadalcázar) & 59 & \\
\hline 1625 & 125 & (Arzáns) & & \\
\hline 1626 & $\sim 60$ & (Cañete) & & *ce vice-roi n'a aucune parenté avec \\
\hline 1633 & 122 & (Juan de Carvajal) & 81 & \\
\hline 1637 & $>100$ & (Alonso Barba) & & \\
\hline 1663 & 130 & (Mendoza ; Calancha) & & N.B. : Voir à la fin de l'article les \\
\hline 1692 & 57 & (vice-roi comte de la Monclova) & & commentaires sur ce tableau. \\
\hline 1693 & 34 & (vice-roi marquis de Cañete)* $^{*}$ & 29 & \\
\hline 1781 & & & & \\
\hline 1787 & $>72$ & (Cañete) & & \\
\hline 1788 & & & 33 & \\
\hline 1790 & & & 33 & \\
\hline
\end{tabular}


dans la mesure du possible le lit d'un cours d'eau intermittent. En décembre 1574, les travaux de creusement de la Ribera commencèrent en même temps que le montage des usines riveraines. Pas moins de deux cents officiers espagnols et de quatre mille manœuvres indiens furent assignés à cette tâche [5]. La Ribera fut inaugurée en mars 1577 soit six mois après l'achèvement des premiers réservoirs. Luis Capoche [4] conte la construction de la Ribera : "Le cours d'eau qui traverse cette ville [Potosi]... ne coule qu'en hiver - espagnol, il considère toujours l'été austral qui est la saison des pluies comme des mois hivernaux - pendant 3 à 4 mois de façon significative. Malgré cela, des usines y furent construites dont la première fut celle du trésorier Diego de Robles Cornejo... Et, comme cela était ingénieux, les hommes bâtirent de forts remparts de 160 varas - 1 vara $84 \mathrm{~cm}-$, de 200 varas et de 300 varas de long et de 8 à 10 varas de large dans des défilés à une demi-lieue de la ville à l'aval des petites plaines où les eaux se concentraient. Les lacs de barrages ont entre 1700 et 1800 varas de circonférence et, en leur centre, 3 estados -1 estado $=160 \mathrm{~cm}-$ de profondeur. Les lacs sont au nombre de 7... Quand l'année est pluvieuse, on peut ainsi raffiner le métal pendant 6 ou 7 mois. Mais il existe actuellement plus d'usines qu'il en est nécessaire et comme ces dernières années ont été sèches, le raffinage a dî se faire en partie sur le cours d'eau permanent à Tarapaya ou en utilisant l'énergie des chevaux". Capoche énumère les usines hydrauliques installées sur la Ribera en 1583 : quarante-neuf (Tableau 2). Puis, il cite Tauacunuño dans le vallon du lac Chalviri où quatre usines utilisaient l'eau de trois lacs. Une autre usine était localisée sur le Rio Chaqui à $40 \mathrm{~km}$ de Potosi. Enfin, vient Tarapaya le site industriel plus ancien avec vingt-quatre implantations.

Néanmoins en ce temps-là, des machines pour concasser les blocs de minerai qui utilisaient l'énergie des hommes et celle des chevaux existaient donc à côté de celles fonctionnant à la houille blanche. Les machines hydrauliques, plus chères mais plus performantes, s'imposèrent progressivement à la fin du XVIe siècle [22].

\subsubsection{La puissance installée (1624-1633)}

Des estimations sont faites pour la puissance installée sur la Ribera. Sachant que les concasseurs étaient animés par une grande roue hydraulique,

a) connaissant la densité de l'eau ;

b) le débit moyen $Q$ du canal $=0,160 \mathrm{~m}^{3} / \mathrm{s}$;

c) la chute d'eau $\mathrm{H}=4,5 \mathrm{~m}$ d'après le diamètre moyen des roues ;

d) et l'efficacité estimée à $75 \%$ pour les roues à aubes qui était le type le plus répandu, on a donc une puissance de :

$\mathrm{P}=\mathrm{gQH}=9,81 \times 0,16 \times 4,5 \times 0,75=5,3 \mathrm{~kW}$

En 1624, soixante-sept concasseurs hydrauliques fonctionnaient sur la Ribera d'où une puissance installée totale de $355 \mathrm{~kW}$ selon Serrano [18]. Ce chiffre peut se pousser jusqu'à $430 \mathrm{~kW}$ parce qu'en 1633 les roues hydrauliques étaient au nombre de quatre-vingt-un. Toutefois à cette dernière date, il faut savoir que les usines ne tournaient pas à plein rendement. En se basant sur un débit moyen de la Ribera un peu plus fort que le précédent soit 250 1/s et un nombre légèrement différent de roues, Rudoph [15] arrive à estimer l'équipement à $450 \mathrm{~kW}$ soit une évaluation compatible avec les nôtres.

\subsection{Arzáns (1705-37) et la décadence (1650-1750)}

Natif de Potosi, Bartolomé Arzáns $(1676-\uparrow 1736)$ était sans doute un modeste maître d'école. Sa chronique [1] va de la création spontanée de la ville en 1545 jusqu'en 1737 car son fils la poursuivit encore pendant une année après son décès. Elle fut écrite avec une constance exemplaire entre 1705 et 1737 mais néanmoins la plus grande partie, celle consacrée aux faits historiques, était rédigée dès 1708 .

\subsubsection{La décadence et sa perception}

Extrêmement pieux, Arzáns fut particulièrement marqué par la catastrophe du 15 mars 1626 : la rupture du barrage de San Ildefonso causant plus de deux mille morts (Annexe 2). Arzáns estima que la ville ne se remit jamais et que cet épisode marque le début de la décadence de la cité. Cañete [3] montre bien le contraire car s'il y eut bien des victimes en mars 1626 la population de Potosi dépassait alors très largement 100000 habitants et la seule "peste" de 1719 tua 20000 des 60000 résidants. Du strict point de vue industriel dès 1633, quelques années après la catastrophe, il y avait plus d'usines sur la Ribera qu'auparavant [15].

Toutefois, Arzáns est représentatif du petit peuple, craintif devant Dieu et gardien de la mémoire des catastrophes. Le bras divin n'oublia pas de punir l'impiété des richissimes habitants de Potosi dans les quatre premières décennies du XVIIIe siècle, la sombre époque où Arzáns rédige sa longue chronique. Il fit s'abattre les calamités climatiques, les épidémies et la décadence économique sur la ville. Par conséquent, dans la seconde moitié du XVIIe siècle et la plus grande partie du XVIIIe siècle, la population de Potosi va diminuer sensiblement. D'environ 160000 vers 1610 , les habitants ne seront plus que 70000 vers 1700 , puis que 40000 après la "peste" de 1719 qui décima le tiers des citadins et enfin 20000 en 1779.

Mais en réalité, l'effondrement des mines et par conséquent de la population de Potosi à partir de 1650 environ s'explique en grande partie par la fin de l'approvisionnement régulier en mercure bon marché venant du Pérou [6]. Le milieu du XVIIe siècle voit le dépassement définitif de Potosi par les mines du Mexique qui devient le grand producteur d'argent des Indes espagnoles.

\subsubsection{Les calamités naturelles}

Le Petit Age Glaciaire se situe entre 1560 et 1850 . Il englobe toute la période coloniale espagnole à Potosi (15451825) car son début est localement fixé par la dendrochronologie à 1520 dans l'Argentine voisine [27]. Dans l'actuel sud bolivien, la période $1560-1641$ à prédominance sèche s'oppose à l'époque suivante $1662-1710$ plus humide [14]. Puis pour l'ensemble des Andes, les années 1720-1860 furent une grande période de sécheresse. La correspondance entre les grandes époques de froidure et les sécheresses andines est connue dans l'histoire [14]. Pour la région de Potosi et de La Plata, l'ancien nom de Sucre, le berceau de l'actuelle Bolivie, c'est une période de décadence sans qu'il existe une relation de cause à effet entre sécheresse et dépression économique. Les quatre décennies du XVIIIe siècle furent particulièrement meurtrières. Le climat dans la région de Potosi fut catastrophique. Entre 1705 et 1742, vingt-quatre années sont mauvaises c'est-à-dire deux sur trois [25]. Les sécheresses furent les calamités les plus communes en 1706, 1712, 1713, $1715,1716,1721,1722,1723,1728,1729,1732$ et $1734[1$, 26]. Mais occasionnellement, il y eut aussi des pluies torrentielles et des gelées. En conclusion, dans l'actuel nord-oues 
argentin qui est le prolongement naturel de la région de Potosi, le XVIIe siècle fut plus humide que le XVIIIe siècle. Ce dernier fut caractérisé par une plus grande instabilité climatique où les sécheresses furent les accidents les plus nombreux et les plus graves [13].

\section{- 3.3. Cañete et le troisième boum minier (1750-1800)}

Publié en 1787, le guide de Pedro Vicente Cañete [3] est très fidèle. Il fut sans doute achevé vers 1780 . Cañete fut un administrateur qui assista le gouverneur de Potosi pendant une trentaine d'années. La fin du XVIIIe siècle est marquée par les réformes du despote éclairé Charles III dont le règne (1759-88) constitue l'ultime effort des Bourbons pour restaurer les colonies espagnoles et pour relancer les mines d'argent.

\subsubsection{Vers un inventaire : barrages, concasseurs et usines}

Le vice-roi Francisco Toledo en 1572 avait ordonné par une cédule royale la construction de dix-huit lacs de barrage. Le chroniqueur Mendoza donne le même chiffre de dix-huit lacs durant la seconde moitié du XVIIe siècle. Vingt lacs de barrages se comptaient en 1780 [3]. Leur nombre en 1799 était de dix-neuf et ils alimentaient aussi soixante-cinq fontaines urbaines selon le rapport de l'ingénieur Mosquera qui restaura le système des lacs entre 1789 et 1800 . À la fin du XIXe siècle, il existe encore environ vingt lacs [8].

A l'inverse de la fin du XVle siècle, quand les roues hydrauliques étaient installées à l'extérieur [19] et l'improvisation régnait à cause de la demande sans cesse plus grande de l'argent, au XVIIIe siècle les usines de Potosi se présentaient comme de petites cités fermées par de puissantes murailles où instruments, bâtiments de travail, entrepôts, maisons et chapelles se jouxtaient (cf. Figs. 2 et 6). Les "ingenios", littéralement ce qui est ingénieux, étaient d'abord les concasseurs puis par extension ce nom fut donné à chacune des cités-usines. On distingue les concasseurs à une "tête" de ceux à deux "têtes". Le nombre des concasseurs installés sur la Ribera et dans la région de Potosi sur d'autres cours d'eau varia beaucoup dans le temps selon le degré d'exploitation des mines et la teneur des filons caractérisée par la baisse des teneurs. Sur la seule Ribera, de deux concasseurs en 1576 , on passa à quarante-neuf en 1582 pour atteindre quatre-vingt-un en 1633 (cf. Tableau 2). Puis, leur nombre baissa : vingt-neuf en 1781 seulement lors du troisième boum de l'argent. Autour de Potosi au sens large, la même tendance se retrouve : quatre-vingt-seize concasseurs en 1582 , plus de cent quarante en 1610 , seulement trentequatre en 1693, année caractéristique de la grande crise entre 1650 et 1750 , et enfin plus de soixante-douze concasseurs en 1787 lors du troisième boum de l'argent [3].

Évoquer Pedro Vicente Cañete permet aussi d'aborder le thème de la "mita" ou encore les travaux forcés des indiens dont il fut l'ultime grand défenseur avant son abolition en 1812.

\subsubsection{Des travaux forcés des indiens}

Vers 1750 à cause surtout d'épidémies, la population andine n'était plus que le quart de celle de 1533, date de la conquête espagnole. De plus, à Potosi, le système de la "mita" était tellement inhumain et dangereux que les indigènes devenaient de plus en plus rares autour de la ville. La "mita" touchait les communautés indiennes riveraines du Lac Titicaca au nord (Pérou actuel) jusqu'à la région de Tarija (frontalière de l'Argentine actuelle) au sud. Les travailleurs étaient mobilisés jusqu'à six cents kilomètres de Potosi. Mais, dans la région voisine de Cochabamba, les métis, les petits commerçants et toutes les catégories exemptes de la "mita" se multiplièrent rapidement [7]. En 1780, les indiens affectés à la "mita" n'étaient que 2880 [3]. Entre 1771 et 1773 à Potosi, cette dernière ne mobilisait plus déjà que 3500 hommes contre les 10460 hommes appelés en 1617 et les 13500 à 15000 vers 1575 [7].

C'est uniquement grâce à cette main d'œuvre gratuite que le troisième boum de l'argent entre 1780 et 1800 aurait eu lieu selon l'historien argentin Enrique Tandeter repris par Larson [7]. Cette affirmation est à tempérer d'abord parce que les travailleurs de la "mita" réellement présents dans les mines du Cerro Rico, d'après les pointages soigneux faits dans chaque exploitation, ne furent jamais très nombreux et ce contingent resta stable entre les XVIe et XVIIIe siècles. D'ailleurs, fait significatif, le roi d'Espagne en $1750 \mathrm{admit}$ que chaque usine aurait vingt indiens de "mita" comme ouvriers et manœuvres au lieu de quarante auparavant [7] et c'est là que le travail forcé jouait le plus grand rôle depuis le début de l'introduction de l'hydraulique. Ensuite avec les réformes de Charles III, l'Espagne envoya de bons administrateurs comme Cañete, des experts internationaux et des missions scientifiques pour réactiver et moderniser les mines. La plus célèbre fut la mission allemande Nordenflycht (1789-90) mais les résistances au changement technologique furent fortes. La nouvelle technique de "barils" apportée par Nordenflycht ne fut pas acceptée par les propriétaires d'usines qui préferrent en rester à la technique traditionnelle du "patio" (cf. Annexe 1) [18]. Mais parallèlement, des écoles des mines se fondèrent à Potosi, la première dès 1757. La plus célèbre fut créée en 1779 par le gouverneur Jorge Escovedo, l'Académie de Métallurgie [17], et elle exista jusqu'au début de la guerre de l'indépendance (1809. 25). Mais au-delà de cette discussion, le troisième boum de l'argent s'appuya également sur la restauration des ouvrages hydrauliques par l'ingénieur Mosquera car la plus faible teneur des filons en argent obligea les usines à triturer de plus fortes quantités de minerai. Tandeter [24] note un accroissement de $40 \%$ de la quantité de "l'outillage" lors du troisième boum.

En résumé, la reprise de l'activité à Potosi resta fragile à la fin du XVIIIe siècle. L'industrie de l'argent était caractérisée par le refus de l'innovation technologique [18], la faiblesse de la main d'œuvre, un approvisionnement en mercure trop lointain et une alimentation en houille blanche déficitaire [16] à cause d'une sécheresse générale dans les Andes entre 1780 et 1820 .

\section{IV — L'EFFACEMENT DE L'ARGENT ET DE L'HYDRAULIQUE (XIXe SIECLE)}

\subsection{Une production quasi-interrompue (1800-25)}

Le début du XIXe siècle est marqué par deux crises aiguës pour la production de l'argent : d'abord en 1800-05, la conjonction de tarissements prolongés de la Ribera avec l'interruption, par les guerres de Napoléon [23], de l'importation du mercure extrait des mines espagnoles; ensuite, la lutte pour l'indépendance de la Bolivie, la plus longue des Amériques (1809-25), qui désorganisera tous les circuits de production.

La population de Potosi atteindra son minimum historique durant cette période très troublée car personne ne vit par 
plaisir à 4000 m d'altitude. En 1807, seules 13700 âmes hantaient les rues de la cité. Quand le libérateur Simón Bolívar la visite en octobre 1825 , Potosi n'est plus qu'une villefantôme avec 10000 habitants soit le seizième de la population du début du XVIIe siècle. La plupart des gens s'étaient enfuis à cause de la longue guerre d'indépendance qui ruina l'activité minière : la "mita" fut abolie en 1812 et Potosi fut tour à tour pillée, prise et perdue par les indépendantistes venus d'Argentine $(1810-11,1813,1815)$ et par les royalistes espagnols.

\subsection{La reprise puis le grand boum de la vapeur (1872-} 1895)

Débutant en 1825 à l'indépendance, la période bolivienne de l'exploitation de l'argent est caractérisée d'abord par un petit boum minier centré sur les années 1840 , le quatrième dans l'histoire [11, 12]. Le maximum de la production fut environ deux fois moindre que celui du troisième boum des années 1750-1800 (Fig. 5). Mais aucune nouvelle construction d'ouvrage hydraulique ne fut nécessaire, production et demande en énergie restant faibles. Une nouvelle machine hydraulique pour améliorer toujours le processus d'amalgamation fut construite par les plus grands producteurs d'argent de ce temps, les frères Ortiz d'origine argentine. Toutefois, encore une fois, le caractère conservateur de la corporation des propriétaires d'usines de Potosi fit échouer cette modernisation [12].

L'arrivée de la vapeur et de l'électricité en 1872 déterminera le cinquième boum, le plus important (cf. Fig. 5). Notamment une nouvelle machine pour frapper la monnaie fonctionna à partir de 1876 mais ce boum n'a plus rien à voir avec la houille blanche. Pour alimenter la population quasi-exclusivement, le système hydraulique colonial fut entretenu avec des moyens limités par Guillermo Schmidt à la fin du XIXe siècle [10]. Il subsistait encore des petits ateliers sur la Ribera où le minerai de contrebande et de faible teneur était trituré mais leur outillage était obsolète. En 1877, quatre-vingt-cinq ateliers étaient recensés mais ils n'étaient plus que soixante-cinq en 1892 [10].

\subsection{La transition argent-étain (fin XIXe-début XXe siècles)}

En 1891, l'adoption du Gold Standard par les États-Unis sonna le glas du rôle de l'argent dans le système monétaire international. La démonétisation de l'argent signifia la fin de la plus grosse partie de la demande. Les mineurs et les entrepreneurs boliviens emmenés par trois grands patrons Simón Patiño, Maurizio Hochschild et Aramayo se tournèrent alors vers l'étain dont la demande fut sans cesse croissante jusqu'à la crise mondiale déclenchée par le krach boursier d'octobre 1929. La production record est celle de 1929 qui atteignit 47100 tonnes. Néanmoins l'étain resta demandé durant et après la crise économique des années trente ; il est indispensable dans la confection du bronze en alliage avec le cuivre. Mais surtout, l'importance de l'étain dans la confection des boîtes de conserve en fera un métal stratégique pour l'approvisionnement des troupes au XXe siècle, marqué par deux grands conflits mondiaux puis par l'affrontement des deux blocs. Le prix de l'étain ne s'effondrera définitivement qu'en 1985 avec la mise en place de l'industrie du recyclage des métaux dans l'économie occidentale qui entraînera sa quasidisparition comme entrée importante du budget bolivien.

\section{V — L'ÉTAIN : LA RESTAURATION DE L'HYDRAULIQUE COLONIALE (1935-36)}

Le travail de William E. Rudolph en 1935-36 [15] qui disposa de huit cents ouvriers visa à restaurer le système de barrages en cascade hérité des espagnols (cf. Tableau 1). Cet ingénieur nord-américain fut contacté par le propriétaire de mines bolivien d'origine allemande, Maurizio Hochschild, l'un des trois magnats de l'étain et le plus riche après Patiño [9]. Le groupe Hochschild fournissait $10 \%$ de la production nationale en 1929 et son directeur était le virtuel patron de Potosi au début du XXe siècle et jusqu'à la nationalisation des mines par la révolution de 1952.

\subsection{Les lacs de barrages}

Vers 1930, six lacs alimentaient encore Potosi mais les traces de nombreux réservoirs de la période coloniale subsistaient, soulignées par des dépôts sédimentaires et chimiques. La population de Potosi était de 35000 habitants en 1934, soit seulement environ le cinquième de celle de l'apogée de la ville au début du XVIIe siècle. Toutefois, les besoins individuels en eau du XXe siècle étaient supérieurs à ceux de la colonie. S'y ajoutait la nécessité pour les sociétés qui exploitaient l'étain de disposer d'eau toute l'année comme pour la métallurgie de l'argent jusqu'en 1872.

William E. Rudolph dut affronter trois grands obstacles lors de la restauration des barrages.

a) Les vannes d'évacuation étaient en très mauvais état et seules deux ne nécessitèrent pas de travaux importants. Taillées à l'origine dans un tronc d'arbre puis ensuite fondues en bronze, les vannes étaient la partie la plus faible de l'ingénierie coloniale.

b) Les infiltrations étaient considérables à travers des remblais. Plein, le réservoir de Chalviri perdait 80 litres/seconde. Des ouvrages avaient des fondations en terrain meuble et ne dépassant pas soixante centimètres. Les deux réponses suivantes furent apportées :

b.a.) de nouveaux barrages qui quelquefois s'appuyèrent sur les anciennes digues pour les lacs Atocha, Candelaria, Llama-Miku et Ulistia avec des fondations d'un mètre dans la roche saine ;

b.b.) à Chalviri dont le lac ne pouvait pas être vidangé, un second et nouveau barrage fut édifié très légèrement à l'aval de celui de la colonie. Puis, l'espace entre les deux fut comblé par du béton pour former une seule digue de $5 \mathrm{~m}$ de large, ennoyant totalement le barrage de l'époque coloniale.

c) La plupart des barrages avaient une crête insuffisamment élevée lors des grandes pluies. L'eau débordait, cascadait d'où la ruine d'un grand nombre d'ouvrages. W.E. Rudolph releva la crête des barrages sans déversoir et il rebâtit les tunnels d'évacuation des eaux des lacs Pisco Cocha et Providencia qui s'étaient effondrés ou colmatés.

Toutefois, tout ne put être restauré. Bien des lacs de la colonie avaient disparu définitivement au XXe siècle. L'un d'entre eux était situé dans le vallon de San Sebastián dans un défilé extrêmement étroit et toute trace en disparut lors d'un éboulement. Un seul lac aurait été aménagé à l'époque coloniale dans la région de San José à la place des trois existant en 1930 (San José, Buenaventura et Llama-Miku) ; légèrement à l'aval subsistent les ruines d'une grande digue qui les aurait certainement ennoyés. Enfin, un problème ne pouvait trouver de solution : la forte sédimentation de certains lacs. Ainsi, celui de San Sebastián avait perdu 
$80000 \mathrm{~m}^{3}$ de sa capacité initiale, remblayé sur une épaisseur de $95 \mathrm{~cm} \mathrm{[15].}$

\subsection{Le réseau d'alimentation}

Le réseau d'alimentation en eau de Potosi du début du XXe siècle n'était plus un héritage espagnol à l'inverse des lacs. Sous la colonie, toutes les eaux des barrages sauf ceux des vallons de Pati-Pati, Huacani, San Ildefenso et Soras se jetaient dans le lac San Sebastián avec un système de petits canaux et des cascades. Puis, l'eau allait à la Ribera, le canal traversant la ville et sur lequel la plupart des usines étaient installées sur une quinzaine de kilomètres [15]. L'eau du lac de San Ildefonso servait à la consommation humaine et elle alimentait avec d'autres sources deux cent quatre-vingt-six fontaines publiques au début du XVIIe siècle [20].

Les ruines de deux anciens canaux issus du lac Chalviri épousent encore les montagnes. Leurs tracés diffèrent du système actuel qui présente des secteurs souterrains et qui quelquefois aussi utilise des conduites en fer. D'autres ruines de petits canaux existent encore près des lacs Ulistia, Muniza et du lac asséché de San Lazaró. Mais quand c'était possible, W.E. Rudolph respecta là aussi les travaux antérieurs. Ainsi, le canal de Soras fut réparé et remis en service alors qu'il était hors d'usage et complètement oublié depuis environ 1860. Tous les petits canaux furent nettoyés et restaurés si nécessaire.

Sans restauration significative depuis 1936, les lacs coloniaux continuent d'alimenter la population et les industries de Potosi avec l'ajout de nouvelles retenues construites au XXe siècle lors du boum de l'étain. En 1992, une douzaine d'usines uniquement utilisaient les eaux de la Ribera dans Potosi mais aujourd'hui une trentaine d'établissements fonctionnent, la forte demande de zinc aidant, qui travaillent aussi des minerais de plomb, d'argent et d'étain.

\section{VI $\square$ CONCLUSION}

Dans leur principe, les machines hydrauliques en bois de Potosi essentiellement conçues à la fin du XVIe siècle démontrèrent leur fiabilité ; moyennant la substitution des pièces d'usure et le remplacement au fil du temps de quelques-uns de leurs composants par des éléments en fer, elles fonctionnaient presque inaltérées dans leur dessin encore à la fin du XIXe siècle. Elles ne devinrent totalement obsolètes qu'en 1872 , début de la modernisation totale de l'industrie minière. Leur grande robustesse et leur facilité de réparation furent un obstacle au changement technique et elles favorisèrent indirectement la décadence de Potosi. Le développement technologique de la fin XVIe siècle et du début du XVIIe siècle ne permit pas que la puissance installée sur la Ribera dépassât $450 \mathrm{~kW}$ et la capacité maximale journalière de trituration ne fut que d'environ 320 tonnes de minerai argentifère. En 1583, une année typique de la période la plus florissante (1576 à 1650), quarante-neuf usines hydrauliques étaient installées sur la seule Ribera, le canal baignant Potosi et l'émissaire de la vingtaine de barrages construits à partir de 1573 . Le chiffre maximal d'usines hydrauliques sur la Ribera fut, semble-t-il, de quatre-vingtneuf, chiffre atteint en 1633 , un peu après l'apogée de la cité coloniale vers 1620 . Le débit moyen de la Ribera, aménagée en 1574-77, était de l'ordre de 160 à $250 \mathrm{l} / \mathrm{s}$ et la chute d'eau de $600 \mathrm{~m}$ tandis que les lacs de barrage avaient une capacité totale d'environ 6 millions de $\mathrm{m}^{3}$. La faible puissance instal- lée $(450 \mathrm{~kW})$ ne doit pas faire sourire. Avec sa houille blanche à laquelle s'ajoutaient au début des machines encore plus rustiques à énergie humaine et animale, Potosi produisit 32000 tonnes d'argent de 1545 à 1825 . Néanmoins et sans nul doute, faut-il finir en évoquant les indiens dont les communautés célébraient le départ forcé jusqu'en 1812 vers Potosi comme une offrande aux divinités de la terre et donc comme un voyage sans retour. Par leur sacrifice, Potosi bâtit également sa prospérité durable.

\section{BIBLIOGRAPHIE}

[1] Bartolomé ARZANS [1705-1737] - Historia de la villa imperial de Potosí. (L. Hanke \& G. Mendoza, éds.), 3 tomes, Brown University, Providence (USA), 1965

[2] Peter J. BAKEWELL - La producción registrada de plata en Potosí 1550-1735. Historia y Cultura (La Paz), n²13, 1988, pp. 3-36.

[3] Pedro V. CAÑETE [1787] - Guía histórica, geográfica, física, civil y legal del gobierno e intendencia de la provincia de Potosí. (A. Alba, ed.), Editorial Potosí, Potosí, 1952.

[4] Luis CAPOCHE [1583] - Relación general de la villa imperial de Potosí. (L. Hanke, éd.), tome 122, Atlas, Madrid, 1959.

[5] Laura ESCOBARI - Las lagunas de Potosí. Arte y Arqueología (La Paz), n8-9, 1982-83, pp. 177-184.

[6] Lewis HANKE - Prologo y notas de la edición de la "Relación general de la villa imperial de Potosí de Luis Capoche". Tome 122, Atlas, Madrid, 1959, pp. 5-68.

[7] Brooke LARSON - Colonialismo y transformación agraria en Bolivia. Cochabamba 1500-1900. Ceres/Hisbol, La Paz, 1992.

[8] R.P. Angelico MARTARELLI - El colegio franciscano de Potosí y sus misiones. Noticias históricas. Tipografía Italiana, Potosi, 1890.

[9] Ismael MONTES DE OCA - Sequía en Potosi. EMUSA, Potosí, 1983.

[10] Modesto OMISTE [1893] - Crónicas potosinas. Distribuidora Cultural Sur, Potosí, 4 éd., tome 1, 1992.

[11] Tristan PLATT - Producción, tecnología y trabajo en la Rivera de Potosí durante la república temprana. in: El Siglo XIX. Bolivia y América Latina (Sucre-1994), Travaux de 1'IFEA, La Paz, t. 102, 1997, pp. 395-435.

[12] Tristan PLATT - Las empresas mineras de los hermanos Ortiz y la construcción de las élites nacionales, Salta y Potosí 1800-80. Actas del Coloquio en Homenaje a C.P. Assadourian, 18-20 marzo 1996, UNAM, México, sous presse.

[13] María del Rosario PRIETO, Roberto HERRERA - Las perturbaciones climáticas de fines del siglo XVIII en el área andina. in: Proyecto NOA (NorOeste Argentino), Junta de Andalucía, Sevilla, $\mathrm{n}^{\circ} 1,1992$, pp. 7-35,

[14] María del Rosario PRIETO, Roberto HERRERA, Patricia DUSSEL Clima y disponibilidad hídrica en el Sur de Bolivia y Norte Oeste Argentino entre 1560 y 1710 . Actas del 3 Simposio Sudamericano "Paleoecología, desarrollo del paisaje y del clima de la diagonal árida sudamericana en el Cuaternario Superior". Bamberg. 1994, sous presse.

[15] William E. RUDOLPH - The lakes of Potosi. The Geographical Review (New York), vol. 26, nº 4, 1936, pp. 529-554.

[16] Eduardo SAGUIER - La penuria de agua, azogue y mano de obra en el origen de la crisis minera colonial. El caso de Potosí a fines del siglo XVIII. Revista Latinoamericana de Historia Economica y Social (Lima) $\mathrm{n}^{\circ} 12,1988$, p. $69-80$.

[17] Carlos SERRANO - Transferencia de tecnología y relaciones de intercambio. Caso de estudio: la amalgamación y las escuelas de minería en la colonia. Revista Metalúrgica (Oruro, Bolivie), n¹3, 1993, pp. 53-56.

[18] Carlos SERRANO - Intercambio tecnológico en la amalgamación entre los centros mineros de América con sus homólogos europeos. Boletín Sociedad Geográfica y de Historia "Potosí", n¹6, 1995, pp. 63-82.

[19] Carlos SERRANO - Un ejemplo de proyecto de reempleo : el ingenio San Marcos. Actas del 2 Congreso Nacional de Metalurgia y Ciencias de los Materiales, Diciembre de 1996, Universidad Técnica de Oruro (UTO), sous presse.

[20] Carlos SERRANO, Julio PELAEZ - Potosí y su sistema hidráulico. Boletín Sociedad Geográfica y de Historia "Potosi", n¹4 \& n¹5, 1991 \& 1992, pp. 44-52 \& 75-93. 
[21] Carlos SERRANO, Julio PELAEZ - Potosí y sus lagunas. Revista de Investigaciones Históricas (Universidad Autónoma Tomás Frías, Potosí), 1995-1997, pp. 14-130.

[22] Carlos SERRANO, Julio PELAEZ - La Ribera de la Vera Cruz de Potosí. Rocas y Minerales (Madrid), vol. 24, n5, 1996, pp. 49-67.

[23] Enrique TANDETER - La crisis de 1800-1805 en el Alto Perú. Data (La Paz), n 1 , 1991, pp. 9-49.

[24] Enrique TANDETER - Los trabajadores mineros y el mercado. in Anuario 1996 (R. Arze et al., éds.), Archivo y Biblioteca Nacionales de Bolivia, Sucre, 1996, pp. 53-68
[25] Enrique TANDETER, Nathan WACHTEL - Precios y producción agraria. Potosí y Charcas en el siglo XVIII. Buenos Aires: CERES, Buenos Aires, 1983.

[26] Edgar VALDA MARTINEZ - El agua y las lagunas en Potosí. Presencia Literaria (La Paz), 9 de Octubre 1988, p. 2

[27] Ricardo VILLALBA - Tree-rings and glacial evidence for the Medieval Warm Epoch and the Little Ice Age in Southern South America. Climatic Change, vol. 30, 1994, pp. 1-15.

\section{Annexes}

\section{Annexe 1. \\ Les procédés d'amalgamation à Potosi [17].}

Le "patio" fut introduit à partir des années 1570 à Potosi. L'amalgamation du minerai d'argent et du mercure était connue en Europe depuis les anciens romains. Le procédé du "patio" (mot espagnol signifiant cour) fut inventé au Mexique en 1555 par Bartolomé de Médina. Il se caractérise par la phase de l'amalgamation entre l'argent, le mercure et d'autres agents comme le chlorure de sodium se faisant dans des bassins carrés et sans agitation mécanique autre que manuelle. Quatre de ces bassins sont dessinés par Arzáns au centre de la figure 6 .

Les "barils" sont toujours une technique basée sur l'amalgamation. Ce procédé fut inventé par le R.P. Alonso Barba de Potosi en 1609 puis largement perfectionné en Europe centrale surtout par le baron von Born au XVIIIe siècle. Les barils ou les tonneaux en bois sont remplis de minerai d'argent, de mercure, de limaille de fer et d'eau. Puis, ils sont attachés à un axe mu par une grande roue hydraulique. Les barils tournent lentement pendant deux heures et ensuite, plus rapidement, pendant vingt heures après un ajout ultérieur de mercure.

Le processus d'amalgamation s'achève en moins de vingtquatre heures dans la métallurgie allemande du début du XIXe siècle. Par contre, quinze jours étaient nécessaires pour la technique du "patio" toujours en vigueur à Potosi jusqu'à la seconde moitié du XIXe siècle, après le refus des industriels locaux d'adopter le procédé des "barils" en 1789-90.

\section{Annexe 2. Le barrage de San Ildefonso et l'inondation catastrophique de 1626}

Après une bonne saison des pluies, le lac de San Ildefonso était plein à ras bord. A cause de l'incurie d'un haut fonctionnaire de la couronne qui ne se préoccupa pas de la montée des eaux et ne fit pas ouvrir à temps les vannes, le lac rompit son barrage le dimanche 15 mars 1626 et se déversa sur la ville. Tout fut consommé en deux heures [5]. Le bilan des dégâts selon Arzáns [1] est plus fort que d'après Cañete [3]. Pour le premier, sur cent vingt-cinq "têtes" d'usines (cf. Fig. 6), seules six ne furent pas détruites. Pour le second, sur cent vingt-deux usines installées à Potosi et Tarapaya, un peu moins de la moitié survécurent à la catastrophe. L'évaluation de Cañete sera préférée car les usines de Tarapaya, à quelques $12-18 \mathrm{~km}$ à l'aval de Potosi, ne durent pas trop souffrir de l'inondation. En tout, les usines s'échelonnaient sur une vingtaine de kilomètres depuis la localité Aguas de Castilla, située juste sous les lacs de San Sebastián et de San Ildefonso, et Tarapaya. Par contre, les deux auteurs, Arzáns et Cañete qui utilisèrent souvent des sources différentes, se rejoignent pour fixer le nombre des pertes humaines à plus de deux mille. Toutefois, le bilan selon Arzáns est encore plus lourd avec 4000 victimes. Il est repris par Rudolph en 1936 [15] mais il semble exagéré pour les historiens contemporains après les travaux des éditeurs scientifiques d'Arzáns comme Gunnar Mendoza et Lewis Hanke en 1965 et Alberto Crespo en 1970. Néanmoins, c'est l'une des catastrophes les plus meurtrières au monde dans l'histoire des accidents de barrages depuis le XVIIe siècle, comparable seulement à celles de South Fork (USA) en 1889, Vaiont (Italie) en 1963 et Machhu II (Inde) en 1979 [A. Goubet, comm. pers.]. Le tableau suivant peut en être brossé :

- Volume du lac : $400000 \mathrm{~m}^{3}$ [15].

- Volume évacué lors de la catastrophe : les deux-tiers de la retenue selon certain auteurs $[1,5]$ soit $270000 \mathrm{~m}^{3}$ mais en fait la totalité du lac [21].

- Heures de la catastrophe : entre 13 heures et 15 heures [3]. - Débit moyen pendant les deux heures d'écoulement torrentiel : $60 \mathrm{~m}^{3} / \mathrm{s}$.

- Caractéristiques du barrage : selon Cañete [3], le barrage n'était constitué "comme pour les autres lacs que d'une paroi de gazon, boue et pierres". Ces matériaux ne sont d'après leur description que des dépôts morainiques remaniés par l'homme [21] et on ne parle ni de mortier ni de chaux dans leur construction. Le barrage de San Ildefonso avait été l'un des cinq premiers exécutés après la visite de Toledo en 1572. Il était déjà achevé en 1576 [1] et les travaux devraient y avoir débuté en 1574. Dans ce vallon dont les moraines frontales étaient détruites par l'érosion, les espagnols durent construire un barrage avec une longue digue [15]. Lorsque Rudolph restaura complètement le barrage en $1935-36$, le nouvel ouvrage ne mesura pas moins de $500 \mathrm{~m}$ de long et, au point le plus haut, la hauteur sur fondation est de $8 \mathrm{~m}$ mais généralement elle est de $5 \mathrm{~m}$. Toutefois, la hauteur de la digue de 1626 était sans doute moindre d'après des éléments de défense de l'époque coloniale encore en place sur le site. Après le désastre, les espagnols firent à San Ilde- 
fonso et à San Sebastián, les deux lacs menaçant la ville, des travaux pour "reconstruire les deux barrages de chaux et de galets avec de profondes fondations de 1,70 m d'épaisseur pour diminuer la crainte de la population..." (Cañete [3], p. 91). Par conséquent, il est sous-entendu que les fondations de l'ouvrage de San Ildefonso étaient très faibles avant la catastrophe.

- Faute humaine : les barrages espagnols étaient construits sans déversoir et donc une surveillance des niveaux de garde était assurée. En cas de trop plein, les vannes au nombre de deux étaient ouvertes faisant office de déversoirs ou bien un passage pour les eaux était à la hâte pratiqué lors de leur montée, comme en 1599 par le Dr. Arias de Ugarte sur ce même lac de San Ildefonso. Ce travail était facilité par la faible hauteur de ce barrage ( $2 \mathrm{~m}$ à $5 \mathrm{~m}$ ). Toutefois en mars 1626 , alors que le barrage de San Ildefonso était complètement plein et malgré les conseils du technicien chargé de la maintenance des lacs, le corregidor ${ }^{1}$ Bartolomé Astete ne fit pas ouvrir les vannes. Alors, le vent fut suffisant pour amorcer le processus de déversement. La vague en débordant fit s'ébouler la digue d'abord vraisemblablement à l'endroit précis où le barrage avait déjà été ouvert volontairement en 1599 et après seulement réparé de façon sommaire.

- Débit de pointe. Un débit maximum instantané de l'ordre de $150 \mathrm{~m}^{3} / \mathrm{s}$ est admissible dans une section mouillée non perturbée jusqu'aux premières usines installées en contrebas des lacs de San Sebastián et San Ildefonso à la localité Aguas de Castilla. La section mouillée est estimée à $50 \mathrm{~m}^{2}$ et la vitesse moyenne du courant à $3 \mathrm{~m} / \mathrm{s}$ [A. Goubet, comm. pers.]. En centre ville, quelques deux kilomètres à l'aval, il y a beaucoup plus d'incertitudes sur le débit de pointe lors de la catastrophe parce que des embâcles et des débâcles se succédèrent du fait de l'occupation totale du lit majeur de la Ribera. La moitié de la ville fut sous l'eau et le lit majeur atteignit en moyenne quatre pâtés de maisons de large et quelquefois même six pâtés [1]. Comme le plan urbain de Potosi est un damier parfait, il est facile de connaître le côté d'un pâté moyen de maisons : entre 50 et $80 \mathrm{~m}$ autour de la Ribera dans le centre historique de la ville.

- Pertes : plus de 2000 morts, 360 maisons d'espagnols détruites et 800 maisons d'indigènes, souvent construites en adobe. Pourquoi autant de victimes ? Deux facteurs expliquent essentiellement l'ampleur de la catastrophe : la précarité de tous les édifices de la ville et la surpopulation de celle-ci caractérisée de plus par la concentration des habitants le long de la Ribera et de ses usines, seules sources de la richesse.

Les usines riveraines de la Ribera étaient déjà au XVIIe siècle de véritables cités ouvrières, pourvues de l'essentiel des services, qui concentraient la main d'oeuvre de façon très intensive. L'habitation moyenne des espagnols les plus

1 Officier royal. A Potosi, il était traditionnellement le gouverneur de la ville. Bartolomé Astete gouverna de 1624 à 1628 . fortunés fut toujours très petite et extrêmement mal faite, conséquence directe de la croissance du type champignon de la ville. Encore à la fin du XVIIIe siècle, le voyageur Concolorcorvo qui parcourut l'Amérique du sud, de Buenos Aires à Lima, et visita toutes les villes sur son chemin souligne l'exiguïté et la précarité des maisons bourgeoises de Potosi peu en rapport avec la richesse tirée des mines. En lisant Arzáns [1], les descriptions d'effondrement de maisons voire d'églises sous les coups... de la pluie ou de la grêle sont très fréquentes et il ne pleut qu'environ $400 \mathrm{~mm} / \mathrm{an}$ en moyenne à Potosi ! Lors de la catastrophe, quelques soixante grandes roues hydrauliques d'un diamètre de 4 à $6 \mathrm{~m}$ et d'un poids de $3 \mathrm{t}$ en moyenne étaient installées à l'extérieur sur la Ribera [19] et, emportées par un courant de plusieurs $\mathrm{m} / \mathrm{s}$, elles durent frapper les maisons comme des béliers. Cañete [3] décrit très bien l'inondation : "l'eau rompit [le barrage] avec tant de forces qu'elle arracha la terre et les pierres, qu'elle engloutit toutes les usines qui furent précipitées sur la ville, qu'elle détruisit le reste des installations de la Ribera, qu'elle mit en pièces les roues, renversa les pilons et les axes des roues, qu'elle abattit les murs, qu'elle submergea les maisons - dans la très grande majorité construites sans étage - et qu'elle les emporta, une fois entrée..."

En 1626, la population de Potosí était d'environ 150000 âmes, d'où une ville surpeuplée dans laquelle les gens s'entassaient. Enfin, le dimanche à 13 heures, l'instant de la catastrophe correspond au moment du repas et de la détente chez soi. L'onde de crue fit effondrer les maisons et, si les habitants purent sortir à temps, elle les emporta franchi le seuil de l'habitation.

\section{Commentaires sur le tableau 2 :}

1) L'estimation de Capoche est différente de celle de Toledo car les critères de cet industriel minier ne sont pas les mêmes que ceux d'un administrateur ou d'un historien. On peut supposer que ces derniers comptaient deux fois plus d'usines car ils comptabilisaient le nombre de "têtes" de chaque établissement, soit fréquemment deux. Au temps de Capoche en 1583, il y avait un peu plus de 100 "têtes" fonctionnant sur la Ribera et à Tarapaya.

2) L'effondrement de l'année 1626 est visible. Il correspond à la catastrophe de mars 1626 qui fit plus de deux mille morts sans compter les dégâts matériels.

3) La baisse du nombre des usines après 1663 est très forte. Malgré la crise, le nombre des usines s'était maintenu très élevé pendant quarante ans alors que la production baissait sensiblement depuis les années $1620-50$; une période d'adaptation était nécessaire pour ajuster moyens et production.

4) La reprise de la fin des années 1780 se poursuivra jusqu'à la fin du XVIIIe siècle avec un troisième petit boum de l'argent. 